\title{
2018 Presidential Address-Society of Surgical Oncology: The Fundamental Difference Between Cancer Treatment and Patient Care
}

\author{
Kelly M. McMasters, MD, PhD \\ Division of Surgical Oncology, The Hiram C. Polk, Jr., MD Department of Surgery, University of Louisville School of \\ Medicine, James Graham Brown Cancer Center, Louisville, KY
}

When I was first elected President of the Society of Surgical Oncology, my initial reaction was one of intense pride, which very quickly gave way to a profound sense of embarrassment and humility as I realized that I was being honored for the hard work and dedication of hundreds of others over the course of a couple of decades. While there is no way to appropriately thank everyone, I want to take a moment to mention a few people who have been especially helpful to me through the course of my career. At Colgate University, the late Dr. Roger Hoffman, and at Rutgers University, Dr. Gordon Macdonald and the late Dr. William Moyle, taught me to be a scientist. At the University of Louisville, Drs. Hiram Polk, Jr., J. David Richardson, Bill Cheadle, Michael Edwards, among many others, taught me to be an academic surgeon. At M.D. Anderson, Drs. Charles Balch and Raphael Pollock, along with Merrick Ross, Doug Evans, Jeff Lee, Fred Ames, Mark Roh, Steve Curley, Peter Pisters, and many others, taught me to be a Surgical Oncologist. Any measure of success that I have enjoyed is certainly due to the outstanding residents, fellows, faculty, and staff at the University of Louisville. The Division of Surgical Oncology: Drs. Rob Martin, Chuck Scoggins, Michael Flynn, Amy Quillo, Nicolas Ajkay, Prejesh Philips, and Michael Egger have been a tremendous team. Many more of you in the audience, too numerous to mention, have helped me in many ways, from the current officers and many past presidents of the SSO, to

(C) Society of Surgical Oncology 2018

First Received: 25 March 2018;

Published Online: 6 April 2018

K. M. McMasters, MD, PhD

e-mail: mcmasters@louisville.edu those of you who have been close colleagues, collaborators, mentors, and friends. I also want to thank Eileen Widmer and all of the SSO staff who continue to make this such a great organization. Finally, I thank my wife, Beth, our sons Austin and Steven, and the rest of my family for allowing me to be who I am and do what I do. They understand what I am about to tell you.

This will not be a conventional Presidential Address. After spending all my career immersed in the analysis of data-I will not show you any data. I will not talk about surgical history, education, or training. I will not talk about basic, translational, or clinical research. I will not talk about cancer epidemiology, prevention, or new therapies. My talk is entitled: "The Fundamental Difference Between Cancer Treatment and Patient Care." If you think they are the same thing, perhaps I have chosen my topic wisely.

Many years ago, a 58-year-old woman presented to my office with obstructive jaundice. Her workup revealed cancer of the head of the pancreas. She underwent a Whipple operation and recovered uneventfully. Her final pathology revealed poorly differentiated adenocarcinoma of the pancreas with negative margins and a single, positive peripancreatic lymph node. She completed a course of 5-fluorouricil-based chemoradiation for a period of 6 weeks. Nine months after her Whipple operation, she developed a small periumbilical incisional hernia. She otherwise felt fine, had no symptoms or problems, and had returned to an active lifestyle. When I performed her ventral hernia repair, I found multiple, small peritoneal nodules, undoubtedly representing carcinomatosis. I repaired the hernia, but did not biopsy the nodules, and did not tell the patient about the operative findings. The patient quickly returned to her normal quality of life. She was always very cheerful, active, and involved with family 
activities. She became one of my favorite patients because of her wonderful attitude and appreciative nature.

I continued to see her regularly in the office with no diagnostic tests or imaging studies. I fully expected her to become symptomatic relatively quickly and questioned her in detail during each office visit. However, she was completely asymptomatic and enjoying an excellent quality of life until she developed another small incisional hernia 22 months from the time of her first hernia repair. At this point, she was found to have small lung metastases. For the next 23 months, up until the time of her death, she received a succession of different chemotherapy regimens, some of which she tolerated better than others, but none of which induced a response.

By failing to tell the patient at the time of her hernia repair that she had peritoneal metastasis, I gave the patient 22 months of excellent quality of life without symptoms or the knowledge that she had recurrent disease. Was this unethical, immoral...illegal? Or was it the right thing to do? I certainly have asked myself these questions many times and have concluded that it was the right thing to do. Most people, however, would criticize this as overly paternalistic and a violation of the patient's rights.

What is a reasonable plan of follow-up for patients with cancers for which early detection of metastatic disease offers no advantage? Certainly there are some types of cancer for which routine surveillance with tumor markers and imaging studies is warranted, because reasonable salvage therapies are available to extend survival with a good quality of life. For example, surveillance CEA levels and CT scans make sense for patients with colorectal cancer, because some patients with hepatic metastasis can undergo potentially curative liver resection. However, what possible benefit can there be for early detection of asymptomatic recurrent pancreatic cancer? We all know that resection of pancreatic adenocarcinoma is almost always a palliative operation anyway; almost all patients will eventually die of the disease. Why do we perform CT scans and blood tests every 3 months? So that we can administer "palliative" chemotherapy before the development of symptoms? Well, you can't palliate a patient who is asymptomatic. I would ask the same question for patients who have undergone resection for hilar cholangiocarcinoma, gastric cancer, esophageal cancer, and any other type of solid tumor that we resect for which there are no effective salvage therapies or for which there is no evidence that early detection of asymptomatic recurrence is any better than waiting until the patients are symptomatic.

I will add one caveat. There is an important distinction between tests to detect actionable local or regional recurrence versus systemic recurrence. Surgeons are locoregional disease control specialists; we sometimes cure patients only by virtue of the fact that some cancers, in some patients, have not progressed beyond the local and regional disease we resect. Failure of locoregional disease control can cause untold misery for our patients, and salvage procedures/treatments when the locoregional recurrence is detected early can sometimes prevent or mitigate this misery. For solid tumors with this type of biology, remember your solemn duty as a surgical oncologist to achieve locoregional disease control when possible.

Q-TWiST analysis (Quality-adjusted Time Without Symptoms of disease or Toxicity) is a validated method for analyzing the impact of cancer therapy on quality, as well as quantity, of life. The idea is that patients may not always value prolongation of life if involves decreased quality of life related to cancer symptoms or toxicity of treatment. Certainly, the best quality of life is that time during which the patient has no symptoms and no knowledge of recurrence. This is the survival time we should strive to prolong. If there are no salvage therapies that offer a reasonable chance of prolonged survival or cure when asymptomatic recurrence is found, why bother to look very hard to find bad news? Most patients have their recurrence detected because of symptoms anyway. False-positive tests, especially in the era of PET/CT scans, can lead to a devastating sequence of unhelpful additional studies, biopsies, and anxiety. Yet, at cancer centers everywhere, there is a kneejerk reaction to order blood tests and imaging studies every 3-6 months for virtually every kind of solid tumor (NCCN guidelines are all over the map). I know that further progress in treatment of metastatic disease relies on patients entering clinical trials of new therapies. Yet, I do not think we would have less accrual to clinical trials if we waited for such patients to become symptomatic.

For patients with cancers for which there is no good rationale for early detection of recurrence, I tell the patient, "We will proceed with the assumption that you are cured of your cancer unless proven otherwise." I don't remember exactly from whom at M.D. Anderson I borrowed this line, but I have found it useful in counseling many patients. I have been amazed to find that almost all patients understand this philosophy and agree to follow this plan. I see these patients in the office regularly, weigh them, do a physical examination, and most importantly, take a little time to talk to them. I ask about their quality of life and activities and may discuss anything from fishing to family matters. Patients know that they should call immediately if they develop any new symptoms or problems between office visits. Decisions about imaging and other tests are driven by symptoms, not by protocol. If the patient is not comfortable with this watchful waiting strategy, we will decide together on a reasonable surveillance plan.

I could be accused of offering false hope for such patients, because many will relapse and die of their 
cancer-but not all. It is hope that sustains these patients. Hope is what allows them to experience the greatest quality of life after treatment of potentially curable cancers, even if that potential is small. I think it is a laudable goal to prolong the time until the patient must face the reality of terminal disease. After all, how can we measure the value of 22 months of quality survival without symptoms or knowledge of recurrence?

Consider the case of my patient, a 75-year-old widower who had just finished chopping wood in February 2010 when he noticed what he thought was blood in his urine. It turned out instead that he had dark urine from obstructive jaundice. He was found to have two separate primary adenocarcinomas: a T2 gallbladder cancer and a T4b duodenal cancer. He underwent segment IVb/V liver resection, portal lymph node dissection, and Whipple operation. Although he had negative margins of resection, he had four positive lymph nodes with extensive extracapsular extension. After considering adjuvant chemotherapy and radiation therapy, he declined to take any additional treatment. In good conscience, not even I could use my line about assuming he was cured of his cancer unless proven otherwise. We had a frank discussion about his prognosis, and he told me he just wanted to enjoy whatever time he had left on this earth. I saw him in the office every 3 months. I never checked any tumor markers or CT scans. Then, every 3 months turned into every 6 months. Then, every 6 months turned into every year. I talked to him a few weeks ago. He said that he is indeed still on this side of the dirt and is still kicking (although maybe not quite as high as he used to). He still does some work around the farm, is healthy and active, and has a new girlfriend. They go dancing every week and play cards with friends and do lots of other stuff (I didn't inquire further about the other stuff). If he has recurrent cancer, he doesn't want to know about it, and neither do I. He is asymptomatic and has an excellent quality of life at 83 years of age.

I was prepared to tell you many other stories of patients like this, but you get the point. As surgical oncologists, we all have patients like this and stories to tell. I know that most of the stories don't end like this, but there are a couple of lessons here. I didn't know how much time my patient had left on this earth, but whether it was 8 months or 8 years, I was determined that he wouldn't spend his remaining time in doctors' offices and at the hospital getting blood tests and CT scans and some unproven adjuvant therapy regimen we would have to invent. I was determined that he would have quality time without symptoms or knowledge of recurrence. He knew to call me at the first sign of any problems. It just turned out that he never needed to call. Furthermore, in this day and age of personalized cancer therapy, targeted agents, immunotherapy, multidrug cytotoxic chemotherapy regimens, and a dozen different ways to deliver radiation therapy, it is easy to forget that that the original cancer therapy is still the most effective treatment for solid tumors. In many cases, even sometimes hopeless cases, a good operation is still the single best thing we can do for our patients.

Multidisciplinary treatment is generally considered to be the best approach to cancer patient care. Most of the time, that is true. Yet how many times have you seen patients with incurable cancer who have had innumerable chemotherapy regimens and radiation therapy administered right up to the time of their demise-when everyone knew these treatments were futile? Lest we start throwing stones from inside our glass house, we need to evaluate critically the value of all that is done in the name of surgical palliation as well. No matter how many times we discuss the issue of futile surgical care at my own departmental morbidity and mortality conference, many surgeons (myself included) continue to fall into this trap.

I am regularly astounded at how frequently patients and their families, yearning for someone to tell them the plain truth, have encountered physicians who have made the truth so elusive. Sometimes what is needed is one good doctor who knows the patient well enough to help make the right decisions. This includes the decision to stop chemotherapy and institute palliative care. For some reason, it is much easier for physicians to switch to futile fourth-line chemotherapy than to tell the patient it is time to stop treatment. Patients are forever grateful if you tell them the truth, especially if you can summon just a touch of compassion when you deliver the bad news.

I remember when I received a thoughtful handmade gift from a patient and his wife, simply for calling them about a CT scan result that demonstrated recurrent, inoperable, and incurable hepatocellular carcinoma 2 years after his left hepatectomy. In the card that accompanied the gift, they expressed their appreciation for the fact that I had called personally in a timely manner-something that, in their experience, was rare for physicians to do. As touched as I was by this gesture of gratitude from a dying patient, it made me reflect on the times I have not always been so diligent in promptly communicating test results. Certainly, all of us can do a little better in this regard. Patients do not always expect miracles, but they appreciate even the smallest signs of concern that are easy for busy clinicians to overlook.

The most challenging, but perhaps most rewarding, part of the job of being a surgical oncologist is to help ease patients over to the other side with dignity. I let my patients with incurable cancer know that if it gets to the point that further therapy will cause more harm than good, I will tell them. When it gets to that point, I do tell them. Many times, patients are relieved and just want someone to give them permission to stop treatment. The available evidence 
suggests that palliative supportive care enhances not only quality, but duration of life. It is imperative that we teach physicians how to initiate appropriate end-of-life discussions well before the end of life.

But, you might protest, the patient and the family want "everything done" to keep fighting the cancer. This is where being a good doctor comes in. I guarantee that no one wants to suffer with toxicity from futile therapy, and no one wants to watch his/her family member go through this. These are the hard conversations you shouldn't avoid. This is where you can make the difference between death with dignity and death with indescribable misery.

Think about the way that people talk about patients with cancer as if they were fighting a war. Patients are "warriors." Patients are always "battling" cancer. They are told to "keep on fighting" the cancer. They are going to "beat" cancer. We have even had a "War on Cancer." Patients who suffer through the toxicity and side effects of treatment without complaint are "courageous." In Jimmy Valvano's iconic speech as he was dying of cancer, he uttered what is now the motto and a registered trademark of the Jimmy V foundation: “Don't give up...don't ever give up!" ${ }^{\circledR}$ Most people take this to mean that cancer patients should never quit taking chemotherapy and other cancer treatments. Well, what happens if you decide to quit "battling" cancer, because further treatment is toxic and without benefit? Does that mean that you are "giving up?" If you complain about side effects and toxicity, are you a coward? If you accept hospice care should you be ashamed because you surrendered? This isn't about fighting a war; it is about appropriate end-of-life cancer care. We expect cancer patients to walk through the fires of Hell and back in order to live for an extra month, yet many of them just suffer the ravages of overly aggressive treatment. The true heroes are not only those who fight to the bitter end knowing that the fight is hopeless; they are those who know when it's time to accept supportive end-of-life care. It is time for us to change the conversation about appropriate care for end-stage cancer patients. Many of you from other countries do a better job of this than we do in the United States; we still have a long way to go.

Forgive me if I seem to be proselytizing about this topic. You see, my family and I know a little bit about it. We know all about toxicity, palliation, and quality of life. As many of you know, my son, Owen, was diagnosed with T cell acute lymphoblastic leukemia (T-ALL) at age 11 . Leukemia is a cowardly blood cancer that surgeons can't resect. Here is Owen prior to his diagnosis-a funny, outgoing, happy-go-lucky boy with an adorably infectious smile. He made friends easily and made everyone laugh. He loved skateboarding and snowboarding and basketball and tennis and video games. He was smarter than the rest of us and had a brilliant future ahead of him.
Pediatric Oncologists can push children to extreme limits of toxicity, because, in general, kids are a lot more resilient than adults. While there is much talk these days about using MELT (most effective least toxic) principles in guiding therapy, I can assure you that Pediatric Oncologists are not afraid of toxicity. The general strategy appears to be to throw every possible active chemotherapeutic agent at these kids in the hope that the child is tougher than the cancer. Sometimes it works; sometimes it does not.

Owen underwent over 4 years of continuous therapy, including innumerable cycles of systemic chemotherapy and intrathecal therapy, neutropenia, and sepsis-countless days and nights in a pediatric hospital. In oncology, we grade toxicity on a scale of 1 to 5 and consider that anything less than about 4 is "acceptable." Considering that grade 5 toxicity is a polite way of saying "dead," I'm not certain that doctors and patients would always agree on what is acceptable toxicity. This is what toxicity looks like when it is your own child.

Every time Owen had a bone marrow biopsy, which was frequently, we would wait on pins and needles until we got the result-the heartbreaking cycle of apparent remission followed by relapse. Eventually, he underwent bone marrow transplantation. His cancer recurred only a few months later. We knew that everything from there on out was palliative treatment, even when we pretended otherwise.

We were able to keep his disease under control for many months. When his cancer became refractory to less toxic treatments, I advocated for liposomal vincristine, because I read a study that showed a meaningful response rate for his type of cancer in this situation. The toxicity was supposedly "acceptable." Here is Owen just before receiving this treatment, when he had a sleepover Christmas party with all his friends at our house that turned into quite a teenage bash. By all accounts, they had a great time.

Here is what liposomal vincristine did to him within a couple of weeks. You see, I did to my own son what I promise my patients I won't let happen to them. I was tempted by the false hope of futile therapy.

So now you have a choice. You can be a Surgical Oncologist who cuts out cancer and delegates the decisions about appropriate follow-up testing and palliative care to others, or you can be a good doctor and do the right thing. You can avoid the difficult conversations, or you can understand the fundamental difference between cancer treatment and patient care.

\section{EPILOGUE}

This audience is filled with those who have devoted their lives to the care of patients with cancer. Also within this audience are those who have, and more importantly, those 
who will perform the innovative research that will relieve suffering, improve quality of life, extend survival, and cure more patients with cancer. The revolution in immunotherapy already has led to a miraculous cure for some patients with previously incurable recurrent B-cell leukemia, melanoma, and other cancers. These miracles only happen because of the single-minded dedication of those who pursue cancer research. To the young people in the audience, the future of our specialty, Owen would want you to be inspired to pursue this dream of hope for patients with cancer. The dream of the day when the most effective cancer treatments will not be measured by their maximally tolerated dose. The War on Cancer will not be won in the operating room; it will be won in the research laboratory and in the clinical trials office. So, don't give up...don't ever give up ${ }^{\circledR}$ - trying to find cures for cancer.

Thank you for allowing me to serve as SSO President. It has been the greatest honor of my career. 